\title{
Iperchilomicronemia: patologia negletta dagli internisti?
}

\author{
Hyperchylomicronemia: a neglected disease?
}

Nel 1967 l'eminente fisiologo americano Donald Fredrickson (1924-2002) propone la prima sistematizzazione moderna di alcune importanti malattie del metabolismo: le iperlipoproteinemie primitive (tabella 1). Tre anni dopo l'Organizzazione Mondiale della Sanità (OMS) fa propria questa classificazione fenotipica (basata, cioè, su caratteristiche fisico-chimiche dei lipidi e del plasma di provenienza), dando inizio a un progredire rapido e affascinante delle conoscenze in tale ambito. Il metabolismo delle lipoproteine ricche in colesterolo (Low Density Lipoproteins, LDL, e High Density Lipoproteins, HDL, in base alle loro caratteristiche di separazione con ultracentrifugazione) viene sempre più strettamente correlato con lo sviluppo di aterosclerosi e con gli eventi cardiovascolari (LDL, ruolo favorente; HDL, ruolo protettivo) e la ricerca ne riceve impulso. In particolare, si definiscono le basi molecolari delle ipercolesterolemie e la loro genetica: l'ipercolesterolemia familiare è posta in relazione con un difetto del recettore LDL, ma si riconoscono poi una forma determinata da una mutazione dell'apolipoproteina B100, ligando di questo recettore, una terza forma dovuta ad alterata degradazione cellulare del recettore stesso e, ancora, una malattia recessiva da anomalie regolatorie [1].

Le malattie del metabolismo delle lipoproteine ricche in trigliceridi (Triglyceride Rich Lipoproteins [TRL]: Very Low Density Lipoproteins [VLDL] e chilomicroni) hanno invece a lungo rappresentato la "Cenerentola" della lipidologia. Con l'eccezione dell'iperlipemia combinata familiare (le cui basi genetiche sfuggono ancora ad approfondite ricerche), esse non sono state correlate con sicurezza allo sviluppo dell'arteriosclerosi. Ciononostante Donald Zilversmit definiva, già nel 1979, l'aterosclerosi come un fenomeno postprandiale, dunque in verosimile rapporto con elevati livelli di TRL [2].

Le iperchilomicronemie (cioè le ipertrigliceridemie caratterizzate da un aumento dei chilomicroni, lipoproteine prodotte dall'intestino per veicolare i lipidi dietetici) rappresentano una causa non frequente di ipertrigliceridemia, ma sicuramente rilevante per i problemi clinici sottostanti [3]. Spesso si appalesano "casualmente" durante una

Tabella 1 Classificazione fenotipica delle dislipidemie.

\begin{tabular}{lllllll}
\hline Fenotipo & Lipoproteine in eccesso & Aspetto del siero & Colesterolo & Trigliceridi & Aterosclerosi & Prevalenza \\
\hline I & Chilomicroni & Surnatante cremoso & $=/ \uparrow$ & $\uparrow \uparrow \uparrow \uparrow$ & $-($ ?) & Molto raro \\
\hline Ila & LDL & Limpido & $\uparrow / \uparrow \uparrow \uparrow$ & $=$ & +++ & Comune \\
\hline IIb & LDL + VLDL & Torbido & $\uparrow / \uparrow \uparrow$ & $\uparrow / \uparrow \uparrow$ & +++ & Comune \\
\hline III & IDL & Torbido & $\uparrow / \uparrow \uparrow$ & $\uparrow / \uparrow \uparrow \uparrow$ & +++ & Raro \\
\hline IV & VLDL & Torbido & $=/ \uparrow$ & $\uparrow / \uparrow \uparrow$ & + & Comune \\
\hline V & VLDL + chilomicroni & Surnatante cremoso, & $\uparrow / \uparrow \uparrow$ & $\uparrow \uparrow \uparrow$ & + & Raro
\end{tabular}

Legenda: $L D L=$ Low Density Lipoproteins; HDL = High Density Lipoproteins; VLDL = Very Low Density Lipoproteins; IDL = Intermediate Density Lipoproteins.

(Fonte: OMS, 1970) 
Tabella 2 Proteine alterate nelle forme familiari di iperchilomicronemia.

\begin{tabular}{lll}
\hline Proteina & Ruolo & Commenti \\
\hline $\begin{array}{l}\text { Lipasi lipoproteica } \\
\text { (LPL) }\end{array}$ & $\begin{array}{l}\text { Enzima responsabile dell'idrolisi } \\
\text { di trigliceridi, VLDL e chilomicroni }\end{array}$ & $\begin{array}{l}\text { Primo difetto molecolare identificato } \\
\text { nell'iperchilomicronemia }\end{array}$ \\
\hline Apo C-II & Attivatore della lipasi lipoproteica & $\begin{array}{l}\text { L'infusione di apo C-II nei soggetti carenti } \\
\text { normalizza il profilo lipidico }\end{array}$ \\
\hline Apo A-V & Proteina adattatrice (si lega a GPIHBP1) & Recentemente identificate famiglie affette \\
\hline GPIHBP1 & "Piattaforma" molecolare per & Recentemente identificate famiglie affette \\
& la lipolisi mediata da LPL & \\
\hline LMF1 & $\begin{array}{l}\text { Fattore coinvolto nella maturazione } \\
\text { intracellulare di LPL in forma attiva }\end{array}$ & Recentemente identificate famiglie affette \\
\hline
\end{tabular}

determinazione di laboratorio e rendono inaffidabile, se non impossibile, il dosaggio di molti parametri ematochimici. Il sangue prelevato appare di colore rosa salmone, il plasma sembra latte, la trigliceridemia è superiore a $1.000 \mathrm{mg} / \mathrm{dL} ; \mathrm{i}$ valori di colesterolo talora possono essere elevati, anche sensibilmente, in rapporto alla presenza concomitante di VLDL o di prodotti di degradazione delle TRL (remnant): è il cosiddetto fenotipo $\mathrm{V}$ (tabella 1$)$.

L'iperchilomicronemia primitiva è una rara malattia prevalentemente infantile, caratterizzata da addome acuto e pancreatite, dovuta a un'attività bassa/assente dell'enzima lipasi lipoproteica in rapporto a varie anomalie oggi in parte chiarite (tabella 2) [4]. Le forme più comuni di iperchilomicronemia sono tuttavia quelle dell'adulto, frequentemente secondarie a scompenso glicemico, in particolare in caso di diabete misconosciuto e/o esacerbato da farmaci. Spesso è presente un'anomalia del metabolismo lipidico sottostante [5], per esempio un difetto eterozigote di lipoprotein-lipasi (frequente nell'adulto) o un'iperlipemia familiare combinata: l'interazione gene-ambiente determina in questi casi l'età di insorgenza e la gravità della malattia.

Le complicanze cliniche dell'iperchilomicronemia (pancreatite acuta, xantomatosi cutanea eruttiva) sono particolarmente frequenti per livelli di trigliceridemia superiori a $2.000 \mathrm{mg} / \mathrm{dL}$ [6], seppure possano imprevedibilmente mancare. In passato l'ipertrigliceridemia era considerata complicanza della pancreatite, ma più modernamente viene interpretata come sua determinante patogenetica: le ampie fluttuazioni della trigliceridemia stessa nell'arco di ore o giorni (anche in ragione del digiuno instaurato) possono tuttavia offuscarne il significato.

Il paziente adulto con sangue "lattescente" dovrebbe ricevere un attento esame obiettivo (ricerca di epato-splenomegalia e xantomatosi eruttiva, esame del fondo dell'occhio alla ricerca della lipemia retinalis) ed essere interrogato sull'utilizzo di farmaci potenzialmente implicati (beta-bloccanti, diuretici, estroprogestinici, cortisonici, resine a scambio ionico, acido retinoico e derivati, interferoni, inibitori delle proteasi, chemioterapici, immunosoppressori ecc.). La determinazione della glicemia 0 , in rapporto alle difficoltà analitiche descritte, della glicosuria (anche con stick urinario) rappresenta un esame fondamentale poiché può indirizzare verso una forma secondaria a scompenso diabetico. L'ulteriore diagnostica di laboratorio è riservata a pochi centri specializzati.

Causa il rischio di pancreatite, l'iperchilomicronemia è l'unica forma di iperlipoproteinemia che richieda un trattamento urgente: la terapia è prevalentemente dietetica (correzione di eventuali eccessi, abolizione di alcol, alimentazione ipolipidica, utilizzo di trigliceridi a media catena) e farmacologica (risultati incostanti da parte di fibrati e acidi grassi omega-3; dubbio il ruolo dell'eparina). Talora può essere impiegata la plasmaferesi (plasma exchange o doppia filtrazione) per periodi brevi o prolungati, ma tale esperienza rimane ancora aneddotica.

\section{Bibliografia}

[1] Vigna GB, Fellin R. Lipoproteine e dislipoproteinemie. In: Enciclopedia Medica Italiana. Aggiornamento Vol III. Firenze: USES, 2006: pp. 1937-48.

[2] Zilversmit DB. Atherogenesis: a postprandial phenomenon. Circulation 1979;60(3):473-85.

[3] Fellin R, Baggio G, Poli A, Augustin J, Baiocchi MR, Baldo G, et al. Familial lipoprotein lipase and apolipoprotein C-II deficiency. Lipoprotein and apoprotein analysis, adipose tissue and hepatic lipoprotein lipase levels in seven patients and their first degree relatives. Atherosclerosis 1983;49(1):55-68.

[4] Hegele RA. Plasma lipoproteins: genetic influences and clinical implications. Nat Rev Genet 2009;10(2):109-21.

[5] Yang T, Pang CP, Tsang MW, Lam CW, Poon PM, Chan LY, et al. Pathogenic mutations of the lipoprotein lipase gene in Chinese patients with hypertriglyceridemic type 2 diabetes. Hum Mutat 2003;21(4):453.

[6] Brunzell JD, Deeb SS. Familial lipoprotein lipase deficiency, apo C-Il deficiency, and hepatic lipase deficiency. In: Scriver CR, Beaudet AL, Sly WS, Valle D, editors. The Metabolic \& Molecular Bases of Inherited Disease. New York, NY: McGraw-Hill; 2001. p. 2789-816.

Giovanni B. Vigna*, Renato Fellin Sezione di Medicina Interna, Gerontologia e Geriatria, Dipartimento di Medicina Clinica e Sperimentale, Università degli Studi di Ferrara

${ }^{*} E$-mail: vgg@unife.it 